\title{
El beneficio del tratamiento antihipertensivo con drogas no está influenciado por el sexo del paciente
}

Effect of antihypertensive drug treatment on cardiovascular outcomes in women and men Gueyffer F, Boutitie F, Boissel JP, et al. Ann Intern Med 1997;126:761-67.

\section{Objetivo}

Cuantificar el efecto del tratamiento con drogas antihipertensivas en ambos sexos y determinar si existen diferencias entre hombres y mujeres.

\section{Fuente y selección de datos}

Se obtuvieron los datos de los pacientes individuales de una base de datos de siete estudios randomizados (INDANA). Estos siete estudios incluyeron 19.975 hombres y 20.802 mujeres. Las drogas utilizadas fueron diuréticos o beta bloqueantes. Se incluyeron los estudios que reportaran los siguientes resultados:1) Accidente cerebrovascular (ACV) fatal; 2) ACV fatales y no fatales; 3) Eventos coronarios fatales; 4) Eventos coronarios mayores fatales y no fatales; 5) Mortalidad por otras causas cardiovasculares (Ej. tromboembolismo de pulmón); 6) Eventos cardiovasculares mayores (suma de los resultados 2,4,5); y 7) Mortalidad total. De todos los estudios realizados que compararon drogas vs placebo en el tratamiento de la hipertensión, se excluyeron 4 que probablemente no habrían alterado las conclusiones debido al poco tiempo de seguimiento en pacientes-año.

\section{Resultados principales \\ En las mujeres, el tratamiento redujo en forma significativa los} ACV fatales y no fatales, OR 0.62 (IC 95\% 0.52-0.73) y los eventos cardiovasculares mayores, OR 0.74 (IC 95\% 0.66-0.83), pero no otros resultados principales. En los hombres, el tratamiento redujo significativamente los siete resultados considerados: Mortalidad global, OR 0.88 (IC 95\% 0.80-0.97); Mortalidad cardiovascular, OR 0.80 (IC 95\% 0.70-0.91); ACV fatal, OR 0.57 (IC 95\% 0.41-0.78); ACV totales, OR 0.66 (IC 95\% 0.56-0.78); Eventos coronarios fatales, OR 0.83 (IC95\% 0.71-0.97); Eventos coronarios mayores OR 0.82 (IC95\% 0.73-0.92) y Eventos cardiovasculares mayores, OR 0.78 (IC95\% 0.71-0.86). No se encontró interacción entre el sexo de los pacientes y el efecto del tratamiento. La reducción del riesgo absoluto (reducción de lầ incidencia) de cualquier evento depende del riesgo basal del paciente y no del sexo. Es decir que si una mujer tiene el mismo riesgo de padecer un evento que un hombre, el beneficio del tratamiento es igual para ambos. Por lo tanto lo que explica la diferencia de OR entre hombres y mujeres es el diferente riesgo que tienen de sufrir un evento y no el hecho de que la respuesta al tratamiento sea diferente por ser hombre o mujer.

\section{Conclusiones}

El beneficio del tratamiento farmacológico de la hipertensión no difiere entre hombres y mujeres en términos de riesgo relativo. La reducción del riesgo absoluto atribuible al tratamiento parece depender del riesgo basal de los pacientes.

\section{Comentario}

Hasta la publicación de este trabajo, no quedaba claro si el efecto del tratamiento antihipertensivo dependía del sexo del paciente. Luego de un reanálisis de varios estudios (1), surgió la posibilidad de que el tratamiento en mujeres jóvenes y blancas podría ser perjudicial. El INDANA ofrece la oportunidad de proveer mayor evidencia del efecto del tratamiento farmacológico antihipertensivo en mujeres. Si bien este estudio incluyó 7 de los 15 trabajos de drogas vs. placebo en hipertensión arterial, los datos representan el $97 \%$ del seguimiento de todos los trabajos. Debemos mencionar que las drogas usadas en los trabajos son diuréticos y beta bloqueantes no pudiendo extrapolarse las conclusiones para otras drogas. Este meta-análisis* no encontró interacción entre el sexo del paciente y el beneficio del tratamiento. Esto signifíca que los efectos del tratamiento serían similares en ambos sexos en términos re- lativos. El beneficio se relaciona con el riesgo basal de padecer un evento vascular. Esto quiere decir que si un hombre y una mujer tiẹnen el mismo riesgo anual de padecer un evento el beneficio del tratamiento sería igual para ambos. La reducción del riesgo absoluto y el número necesario para tratar (NNT) son las mejores estimaciones del beneficio del tratamiento. El efecto de una droga que reduzca el riesgo de $\mathrm{ACV}$ en un $50 \%$ (reducción de riesgo relativo) es muy distinto según el riesgo basal del paciente. Si la incidencia basal es del $1 \%$, la reduce a un $0.5 \%$, con un NNT de 200; en cambio si la incidencia basal es de1 $10 \%$, la reduce al $5 \%$, con un mejor NNT de 20. Debido a la mayor incidencia de eventos en el sexo masculino, el impacto del tratamiento será mayor en los hombres con un NNT menor. Aunque los estudios incluidos en el meta-análisis no fueron diseñados para evidenciar diferencias entre sexos, parece claro que el tratamiento farmacológico es tan efectivo en hombres como en mujeres. Por lo tanto para iniciar tratamiento farmacológico racional e individualizado se debe evaluar con precisión el riesgo cardiovascular del paciente (ver comentario en este número de EVIDENCIA del reporte del VI Joint National Committee de EE.UU).

*Ver glosario

Dra Karin Kopitowski Unidad de Medicina Familiar y Preventiva Hospital Italiano de Buenos Aires

\section{Referencias}

1.Anastos K, Charney P, Charon RA et al. Hy pertension in women: what is really known? The women's Caucus working group on women's health of the Society of General internal Medicine. Ann Intern Med 1991;115:287-93. 